\title{
PERIODIC FLOW OF A SECOND GRADE FLUID DUE TO THE DISKS EXECUTING NON-TORSIONAL OSCILLATIONS IN AN ORTHOGONAL RHEOMETER UNDER THE INFLUENCE OF A MAGNETIC FIELD
}

\author{
H. Volkan ERSOY \\ Department of Mechanical Engineering, Yildiz Technical University \\ 34349 Istanbul, TURKEY \\ E-mail: ersoyhv@yahoo.com
}

\begin{abstract}
The present paper studies the periodic flow of a second grade fluid generated by non-torsional oscillations of the disks rotating in the eccentric form under the application of a magnetic field. Subsequent to the rotational motion of the disks at a common angular velocity about two vertical axes, they perform oscillations horizontally in a symmetrical manner. The exact analytical solutions are derived for both the velocity field and the tangential force per unit area exerted on one of the disks by the fluid. Special attention is paid to the influence of the applied magnetic field and it is investigated how the magnetic field controls the flow when the frequency of oscillation is less than or equal to or greater than the angular velocity of the disks. It is found that the application of the magnetic field leads to thinner boundary layers developed on the disks and the changes in the values of the shear stress components which represent the tangential force exerted on the disks occur at larger amplitude.
\end{abstract}

Key words: magnetohydrodynamics, non-torsional oscillation, orthogonal rheometer, periodic flow, second grade fluid.

\section{Introduction}

Maxwell and Chartoff [1] introduced the Maxwell orthogonal rheometer that simply consists of two rotating non-coaxial disks to study the behaviour of polymer melts. Abbott and Walters [2] were the first to present the solution for the flow of a classical viscous fluid in this rheometer. The flow in the case of a second grade fluid was investigated by Rajagopal and Gupta [3]. Rajagopal [4] proved that the flow in this instrument shows a special motion. Rao and Rao [5] studied the flow of a second grade fluid in the presence of a magnetic field and obtained an exact solution to the problem by means of a cylindrical coordinate system. The reader is advised to read the papers by Rajagopal [6], Srinivasa [7], Siddiqui et al. [8] regarding detailed analysis of various results on this subject.

Unsteady flows of a Newtonian fluid due to the disks performing non-torsional oscillations in the orthogonal rheometer have drawn the attention of several researchers. Erdoğan [9, 10] analysed the nonsymmetrical time-dependent flows resulting from the oscillations of the disks which are initially in the case of concentric rotation. Ersoy [11] investigated the time-dependent symmetrical flow induced by the disks executing oscillations in a symmetrical manner while the two disks are initially rotating about two distinct axes. The impact of a magnetic field in the same configuration was taken into account by Giri et al. [12]. Ersoy [13] studied the periodic flow due to the symmetric oscillations of two porous disks while the disks are originally rotating in the eccentric form. Further, Ersoy [14] analyzed the periodic flow under the influence of an imposed magnetic field in this flow geometry. Apart from the Newtonian fluid, Ersoy [15] studied the periodic flow of a second grade fluid resulting from the oscillations of two disks symmetrically while the disks are originally rotating about two parallel vertical axes. In this paper, the study in Ersoy [15] is extended to the flow occurred by an applied magnetic field. While the two disks are initially rotating eccentrically in the presence of a magnetic field, the periodic flow of a second grade fluid resulting from the disks performing non-torsional oscillations horizontally in the symmetrical form is studied. The behaviour of dilute polymer 
solutions can be modelled by the constitutive equation of second grade fluids. Our main purpose is to study the influence of a magnetic field on these types of fluids in the periodic flow between the disks rotating in the eccentric manner. The physical interpretation of the influence of the magnetic field is discussed with the help of graphs.

\section{Mathematical formulation}

The flow is confined by two insulated disks placed at $z=0$ and $z=h$. The fixed $z^{\prime}$ and $z^{\prime \prime}-$ axes represent the axes of rotation of the upper and lower disks rotating at the common angular velocity $\Omega$. The axes of rotation of the disks separated by a distance $a$ lie on the $y z$ - plane. The region is filled with an incompressible second grade fluid and a uniform magnetic induction $\boldsymbol{B}_{0}$ is applied in the $z$-direction. It is assumed that the induced magnetic field is neglected in comparison to the applied one. Subsequent to the initial motion, the upper and lower disks begin to make non-torsional oscillations as seen in Fig.1. After the nontorsional oscillation motion is initiated, the flow corresponding to the steady-state solution in the fluid is investigated. The schematic diagram of the flow problem is presented in Fig.1.

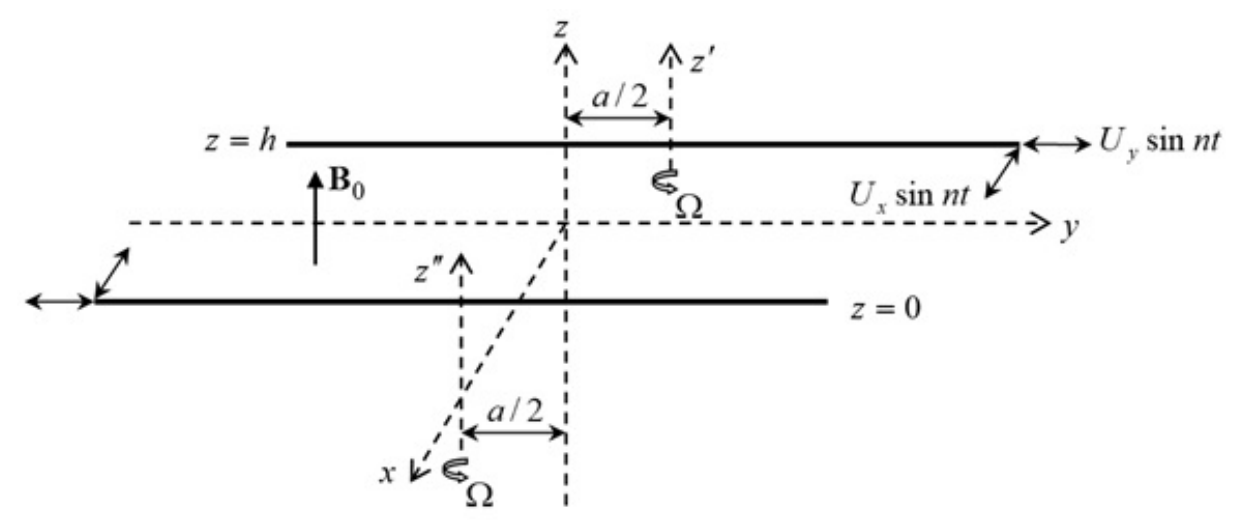

Fig.1. Flow configuration.

The continuity and momentum equations are

$$
\begin{aligned}
& \nabla \cdot \boldsymbol{v}=0, \\
& \rho \frac{D \boldsymbol{v}}{D t}=\nabla \cdot \boldsymbol{T}+\boldsymbol{J} \times \boldsymbol{B} .
\end{aligned}
$$

The Cauchy stress tensor $\boldsymbol{T}$ for a second grade fluid is given by Rivlin and Ericksen [16]

$$
\boldsymbol{T}=-p \boldsymbol{I}+\mu \boldsymbol{A}_{1}+\alpha_{1} \boldsymbol{A}_{2}+\alpha_{2} \boldsymbol{A}_{1}^{2}
$$

The first and second Rivlin-Ericksen tensors are defined as

$$
\boldsymbol{A}_{1}=\operatorname{grad} \boldsymbol{v}+(\operatorname{grad} v)^{T}, \quad \boldsymbol{A}_{2}=\frac{d \boldsymbol{A}_{1}}{d t}+\boldsymbol{A}_{l}(\operatorname{grad} \boldsymbol{v})+(\operatorname{grad} \boldsymbol{v})^{T} \boldsymbol{A}_{l}
$$

The coefficients $\mu, \alpha_{1}$ and $\alpha_{2}$ must satisfy $\mu>0, \alpha_{1} \geq 0, \alpha_{1}+\alpha_{2}=0$ (Dunn and Fosdick [17], Dunn and Rajagopal [18], Fosdick and Rajagopal [19]). 
Thus, the relevant initial and boundary conditions for the problem are

$$
\begin{aligned}
& u=-\Omega(y-\hat{g}(z)), \quad v=\Omega(x-\hat{f}(z)) \quad \text { at } \quad t=0 \quad \text { for } \quad 0 \leq z \leq h, \\
& u=-\Omega(y-a / 2)+U_{x} \sin n t, \quad v=\Omega x+U_{y} \sin n t \quad \text { at } \quad z=h \text { for } t \geq 0, \\
& u=-\Omega(y+a / 2)-U_{x} \sin n t, \quad v=\Omega x-U_{y} \sin n t \quad \text { at } \quad z=0 \text { for } t \geq 0, \\
& u=-\Omega y, \quad v=\Omega x \quad \text { at } \quad z=h / 2 \quad \text { for } \quad t \geq 0
\end{aligned}
$$

where $\hat{f}(z)$ and $\hat{g}(z)$ characterize the location of the centres of rotation along the $z$ - direction for the steady flow of a second grade fluid between the disks rotating in the eccentric form when a magnetic field is present. In the light of Eqs (2.5)-(2.8), we consider a velocity distribution of the form

$$
u=-\Omega(y-g(z, t)), \quad v=\Omega(x-f(z, t)), \quad w=0
$$

which satisfy the continuity equation.

From $\boldsymbol{J}=\sigma(\boldsymbol{E}+\boldsymbol{v} \times \boldsymbol{B})$, the rectangular components of the current density are obtained as $J_{x}=\sigma\left(E_{x}+v B_{0}\right), J_{y}=\sigma\left(E_{y}-u B_{0}\right), J_{z}=\sigma E_{z}$. Since the disks are insulated, $J_{z}$ and $E_{z}$ are found to be zero. Using $\nabla \times \boldsymbol{E}=-\frac{\partial \boldsymbol{B}}{\partial t}$, we find that the $x$ - and $y$-components of the electric field are independent of $z$. Therefore, we have

$$
\begin{aligned}
& \alpha_{1}\left(\frac{\partial^{3} g}{\partial z^{2} \partial t}-\Omega \frac{\partial^{2} f}{\partial z^{2}}\right)+\mu \frac{\partial^{2} g}{\partial z^{2}}-\rho\left(\frac{\partial g}{\partial t}+\Omega f\right)-\sigma B_{0}^{2} g=C_{l}(t) \\
& -\alpha_{1}\left(\frac{\partial^{3} f}{\partial z^{2} \partial t}+\Omega \frac{\partial^{2} g}{\partial z^{2}}\right)-\mu \frac{\partial^{2} f}{\partial z^{2}}+\rho\left(\frac{\partial f}{\partial t}-\Omega g\right)+\sigma B_{0}^{2} f=C_{2}(t) .
\end{aligned}
$$

Let us now introduce the dimensionless quantities as

$$
\begin{aligned}
& \bar{F}(\zeta, \tau)=\frac{f(z, t)+i g(z, t)}{a}, \quad \zeta=\frac{z}{h}, \quad \beta=\frac{\alpha_{1} \Omega}{\mu}, \quad R=\frac{\rho \Omega h^{2}}{\mu}, \\
& M=\sqrt{\frac{\sigma}{\mu}} B_{0} h, \quad V_{x}=\frac{U_{x}}{\Omega a}, \quad V_{y}=\frac{U_{y}}{\Omega a}, \quad \tau=\Omega t, \quad k=\frac{n}{\Omega} .
\end{aligned}
$$

Thus, Eqs (2.10)-(2.11) reduce to the following dimensionless form:

$$
\beta \frac{\partial^{3} \bar{F}}{\partial \zeta^{2} \partial \tau}+(1-i \beta) \frac{\partial^{2} \bar{F}}{\partial \zeta^{2}}-R \frac{\partial \bar{F}}{\partial \tau}-\left(M^{2}+i R\right) \bar{F}=0
$$


with

$$
\begin{aligned}
& \bar{F}(\zeta, 0)=\frac{\hat{f}(z)+i \hat{g}(z)}{a} \\
& \bar{F}(1, \tau)=-V_{y} \sin k \tau+i\left(\frac{1}{2}+V_{x} \sin k \tau\right), \\
& \bar{F}(0, \tau)=V_{y} \sin k \tau-i\left(\frac{1}{2}+V_{x} \sin k \tau\right), \\
& \bar{F}\left(\frac{1}{2}, \tau\right)=0
\end{aligned}
$$

The Poiseuille-type pressure gradient is zero for every value of time because of the symmetric condition expressed by Eqs (2.8) and (2.17); as a result of this, we find $C_{l}(t)=C_{2}(t)=0$.

\section{Periodic solution}

Since we seek a solution that represents a periodic motion of the fluid, it is convenient to recommend a solution in the form

$$
\bar{F}(\zeta, \tau)=\bar{F}_{0}(\zeta)+\bar{F}_{1}(\zeta) \cos k \tau+\bar{F}_{2}(\zeta) \sin k \tau
$$

where

$$
\bar{F}_{0}(\zeta)=\frac{\hat{f}(z)+i \hat{g}(z)}{a}
$$

Substituting Eq.(3.1) into Eq.(2.13), we get

$$
\begin{aligned}
& (1-i \beta) \bar{F}_{0}^{\prime \prime}-\left(M^{2}+i R\right) \bar{F}_{0}=0 \\
& (1-i \beta) \bar{F}_{1}^{\prime \prime}+k \beta \bar{F}_{2}^{\prime \prime}-k R \bar{F}_{2}-\left(M^{2}+i R\right) \bar{F}_{1}=0 \\
& (1-i \beta) \bar{F}_{2}^{\prime \prime}-k \beta \bar{F}_{1}^{\prime \prime}+k R \bar{F}_{1}-\left(M^{2}+i R\right) \bar{F}_{2}=0
\end{aligned}
$$

with

$$
\begin{array}{lll}
\bar{F}_{0}(l)=i \frac{1}{2}, & \bar{F}_{1}(1)=0, & \bar{F}_{2}(l)=-V_{y}+i V_{x}, \\
\bar{F}_{0}(0)=-i \frac{1}{2}, & \bar{F}_{1}(0)=0, & \bar{F}_{2}(0)=V_{y}-i V_{x} .
\end{array}
$$


The solution of Eqs (3.2)-(3.4) subject to Eq.(3.5) is

$$
\begin{aligned}
& \bar{F}(\zeta, \tau)=\frac{i}{2}(\bar{P} \sinh P \zeta-\cosh P \zeta)+ \\
& +\frac{V}{2}(\bar{D} \sinh D \zeta-\cosh D \zeta-\bar{B} \sinh B \zeta+\cosh B \zeta) \cos k \tau+ \\
& +\frac{V i}{2}(\bar{B} \sinh B \zeta-\cosh B \zeta+\bar{D} \sinh D \zeta-\cosh D \zeta) \sin k \tau
\end{aligned}
$$

where

$$
\begin{aligned}
& P=\sqrt{\frac{M^{2}+i R}{1-i \beta}}, \quad B=\sqrt{\frac{M^{2}+i R(1-k)}{1-i \beta(1+k)}}, \quad D=\sqrt{\frac{M^{2}+i R(1+k)}{1-i \beta(1-k)}}, \\
& \bar{P}=\frac{1+\cosh P}{\sinh P}, \quad \bar{D}=\frac{1+\cosh D}{\sinh D}, \quad \bar{B}=\frac{1+\cosh B}{\sinh B}, \quad V=V_{x}+i V_{y} .
\end{aligned}
$$

By means of this solution, the locus of the centres of rotation for the appropriate parameters is revealed in Figs 2-4.

(a)

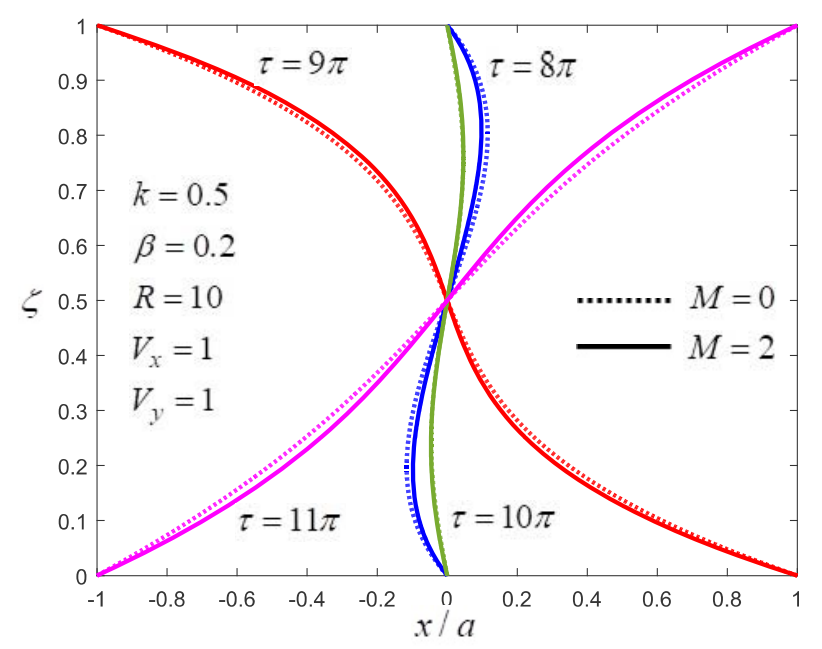

(b)

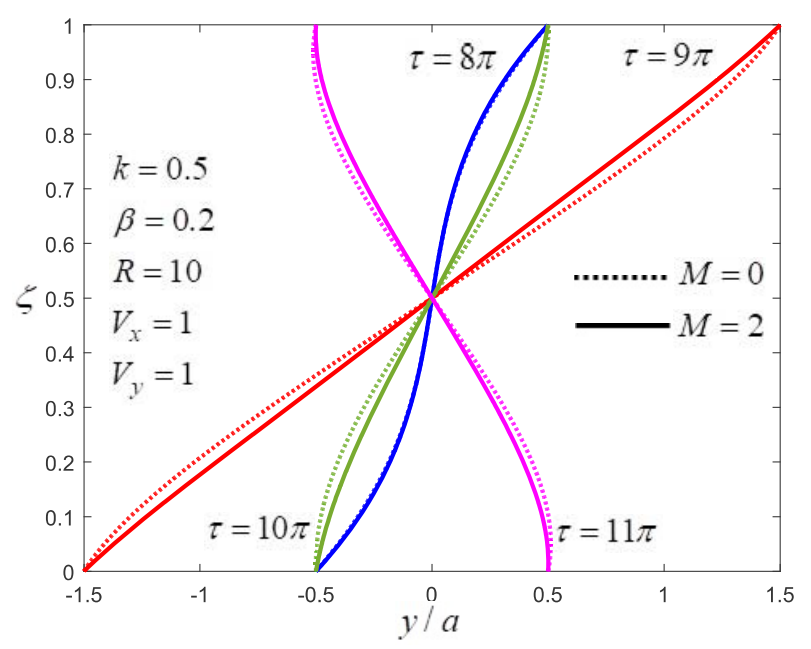

Fig.2. Location of the points where the velocity is zero for $k=0.5$ and other appropriate parameters.

Since the shear stress components $T_{x z}$ and $T_{y z}$ for a second grade fluid are

$$
\begin{aligned}
& T_{x z}=\mu \Omega \frac{\partial g}{\partial z}+\alpha_{1} \Omega\left(\frac{\partial^{2} g}{\partial z \partial t}-\Omega \frac{\partial f}{\partial z}\right) \\
& T_{y z}=-\mu \Omega \frac{\partial f}{\partial z}-\alpha_{1} \Omega\left(\frac{\partial^{2} f}{\partial z \partial t}+\Omega \frac{\partial g}{\partial z}\right)
\end{aligned}
$$


the complex form of the dimensionless shear stress components in the fluid is found as follows:

$$
\begin{aligned}
& \bar{T}_{x z}+i \bar{T}_{y z}=\frac{1}{2}(1-i \beta) K_{1}(\zeta)+ \\
& +\frac{V}{2}\left[-(\beta+i) K_{2}(\zeta)+k \beta K_{3}(\zeta)\right] \cos k \tau+ \\
& +\frac{V}{2}\left[(1-i \beta) K_{3}(\zeta)+i k \beta K_{2}(\zeta)\right] \sin k \tau .
\end{aligned}
$$

(a)

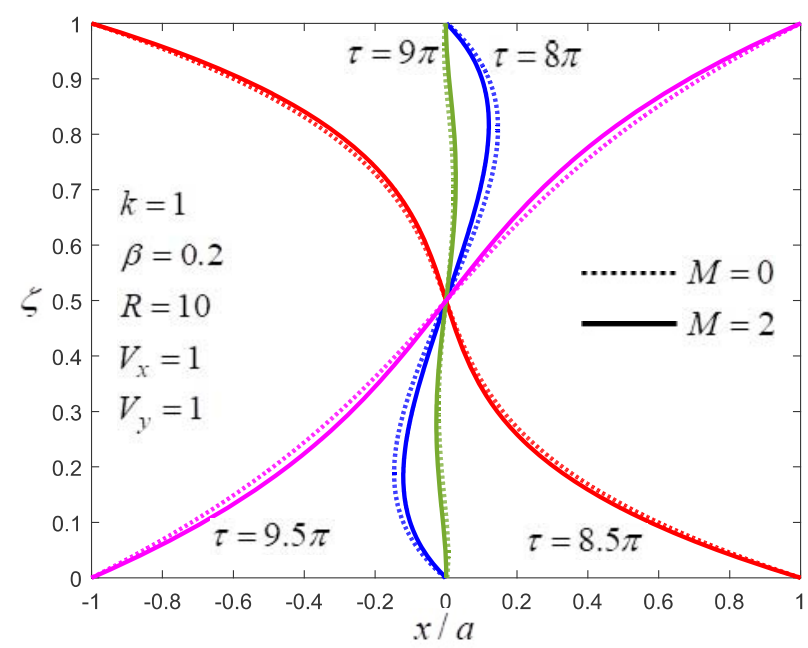

(b)

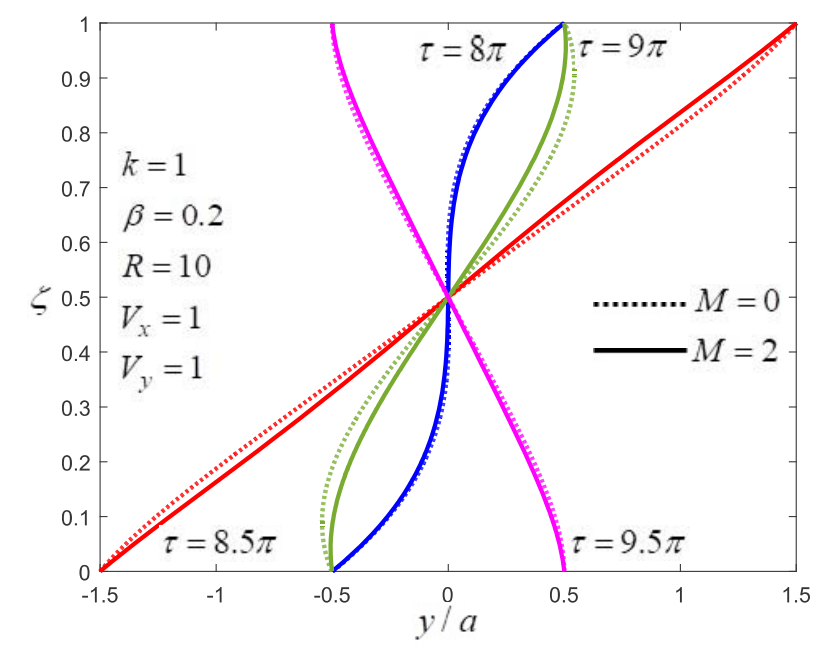

Fig.3. Location of the points where the velocity is zero for $k=1$ and other appropriate parameters.

(a)

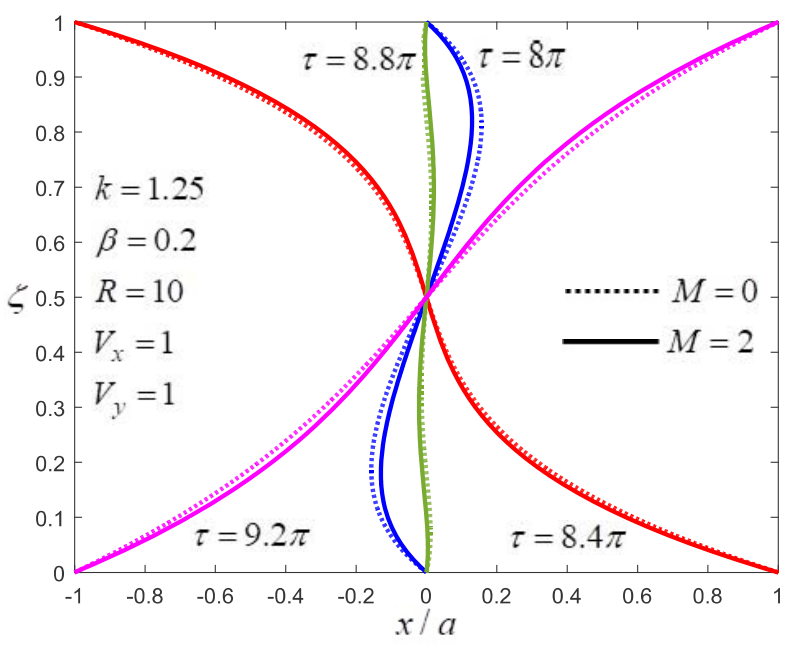

(b)

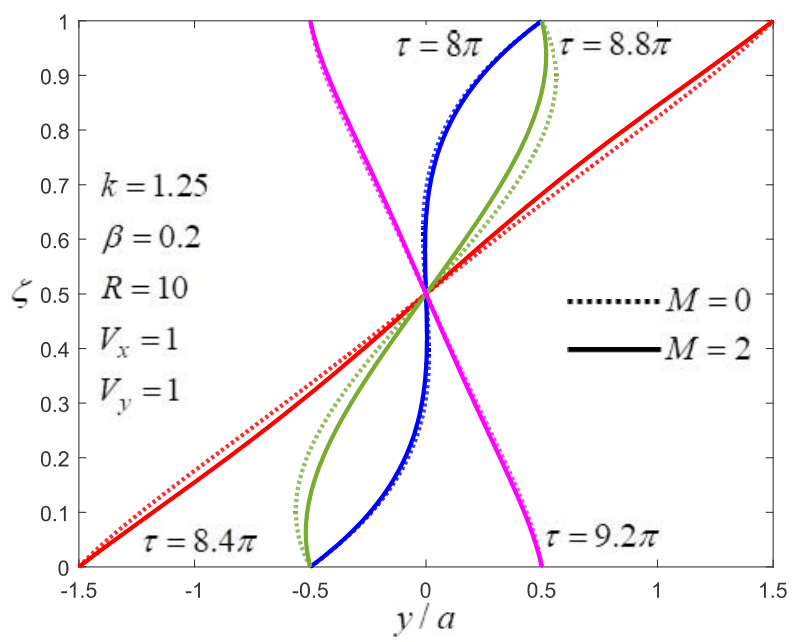

Fig.4. Location of the points where the velocity is zero for $k=1.25$ and other appropriate parameters. 
where

$$
\begin{aligned}
& \bar{T}_{x z}=\frac{T_{x z}}{\mu \Omega a / h}, \quad \bar{T}_{y z}=\frac{T_{y z}}{\mu \Omega a / h}, \quad K_{1}(\zeta)=P H_{1}(\zeta), \\
& K_{2}(\zeta)=D H_{3}(\zeta)-B H_{2}(\zeta), \quad K_{3}(\zeta)=B H_{2}(\zeta)+D H_{3}(\zeta), \\
& H_{1}(\zeta)=\bar{P} \cosh P \zeta-\sinh P \zeta, \quad H_{2}(\zeta)=\bar{B} \cosh B \zeta-\sinh B \zeta, \\
& H_{3}(\zeta)=\bar{D} \cosh D \zeta-\sinh D \zeta .
\end{aligned}
$$

The variation of $\bar{T}_{x z}$ and $\bar{T}_{y z}$ with the appropriate parameters is shown in Fig.5.

(a)

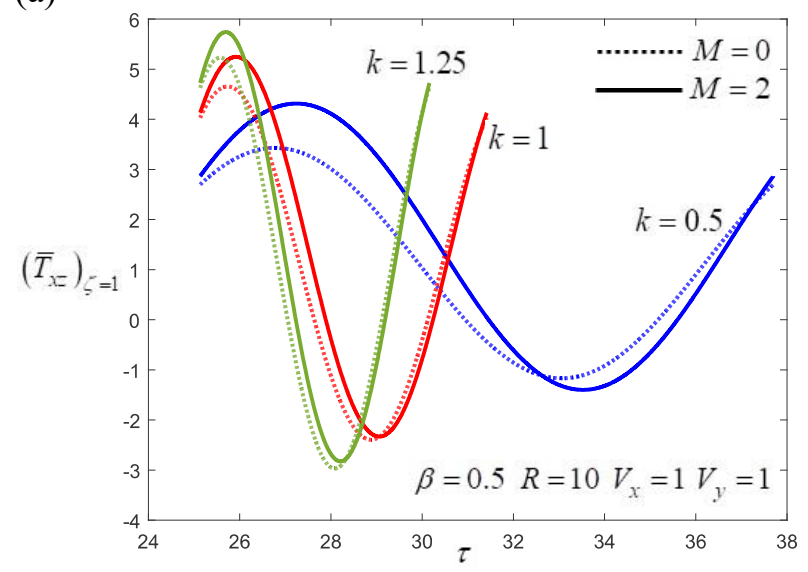

(b)

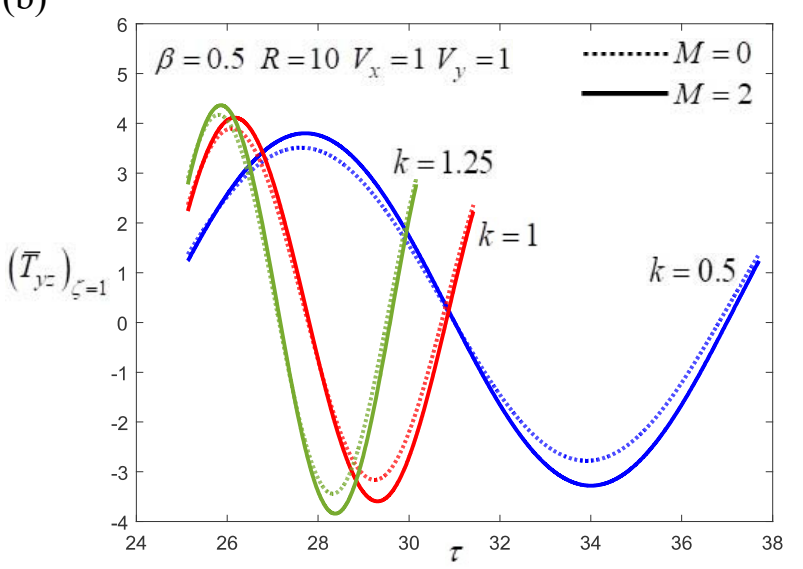

Fig.5. Effect of the magnetic field on $\left(\bar{T}_{x z}\right)_{\zeta=1}$ and $\left(\bar{T}_{y z}\right)_{\zeta=1}$ for the appropriate parameters.

\section{Results and discussion}

The present paper considers the magnetohydrodynamic periodic flow of a second grade fluid due to the non-torsional oscillations of the disks rotating eccentrically. While the disks are initially rotating at the same angular velocity in an eccentric manner in the presence of a magnetic field, the periodic flow of fluid caused by the disks performing oscillations horizontally in the symmetric form is studied. After the oscillation of the disks is started, the flow contains transients at initial times. As the time elapses, the initial transients decay and the flow reaches a periodic state. The periodic state described by the steady-state solutions is considered here. The periodic flow is taken into account after the dimensionless time $\tau=8 \pi$ since the periodicity of the flow already starts at the instant considered for the values of parameters used in the paper (Ersoy [13], Ersoy [14], Ersoy [15]).

In this flow, each $z=$ constant plane rotates about the centres of rotation of the fluid layers which are expressed by $x=f(z, t)$ and $y=g(z, t)$. Since the disks undergo non-torsional oscillations, the location of the centres of rotation varies continuously with time. It is clear that the velocity field and shear stresses depend on all the parameters governing the flow. Since Ersoy [15] analysed this flow in the absence of a magnetic field, the attention in the present paper is focused on the influence of the magnetic field. Figures 2-4 illustrate the coordinates of the centres of rotation for various appropriate parameters when the magnetic field is absent 
and present. In Figs $2 \mathrm{a}$ and $2 \mathrm{~b}$, the frequency of oscillation $n$ is less than the angular velocity of the disks $\Omega$. Figures $3 \mathrm{a}$ and $3 \mathrm{~b}$ show the situation where $n$ is equal to $\Omega$. Figures $4 \mathrm{a}$ and $4 \mathrm{~b}$ display the case when $n$ is greater than $\Omega$. From these figures, it is evident that the motion has a period of $\tau=2 \pi / k$, and the centres of rotation in the mid region get closer to each other when the Hartmann number increases.

An orthogonal rheometer is mainly used to measure the material moduli characterizing non-Newtonian fluids, the calculation of tangential force exerted on one of the disks is especially crucial. When the applied magnetic field is present and absent, the dependence of $\left(\bar{T}_{x z}\right)_{\zeta=1}$ and $\left(\bar{T}_{y z}\right)_{\zeta=1}$ which symbolize the $x$ - and $y$ -components of the non-dimensional force per unit area applied by the upper disk on the fluid, respectively, on the dimensionless time is plotted in Fig.5.

It should be noted that the shear stresses $T_{x z}$ and $T_{y z}$ on the upper disk are mutually equal to those on the lower disk, i.e., $\left(T_{x z}\right)_{z=h}=\left(T_{x z}\right)_{z=0}$ and $\left(T_{y z}\right)_{z=h}=\left(T_{y z}\right)_{z=0}$ as a consequence of the symmetric condition. As clearly seen from Fig.5., the increase of the Hartmann number leads to a rise in the magnitude of the force in the $x$-direction exerted on the disks.

\section{Conclusion}

In this paper, the attention is paid to the analysis of the impact of a magnetic field. The exact analytical solution to the present problem is obtained. It is verified that the fluid motion is retarded due to the effect of the imposed magnetic field. The centres of rotation of the fluid layers in the core region tend to align in the $z$-axis with an increase of the exerted magnetic field. Consequently, the boundary layer thickness decreases and the dimensionless shear stress components on the disks have larger changes with the effect of the magnetic field. When the frequency of oscillation is greater than the angular velocity of the disks, the amplitudes of the non-dimensional shear stresses on the disks become larger. When $n$ is less than $\Omega$, the influence of the magnetic field is more prominent.

\section{Nomenclature}

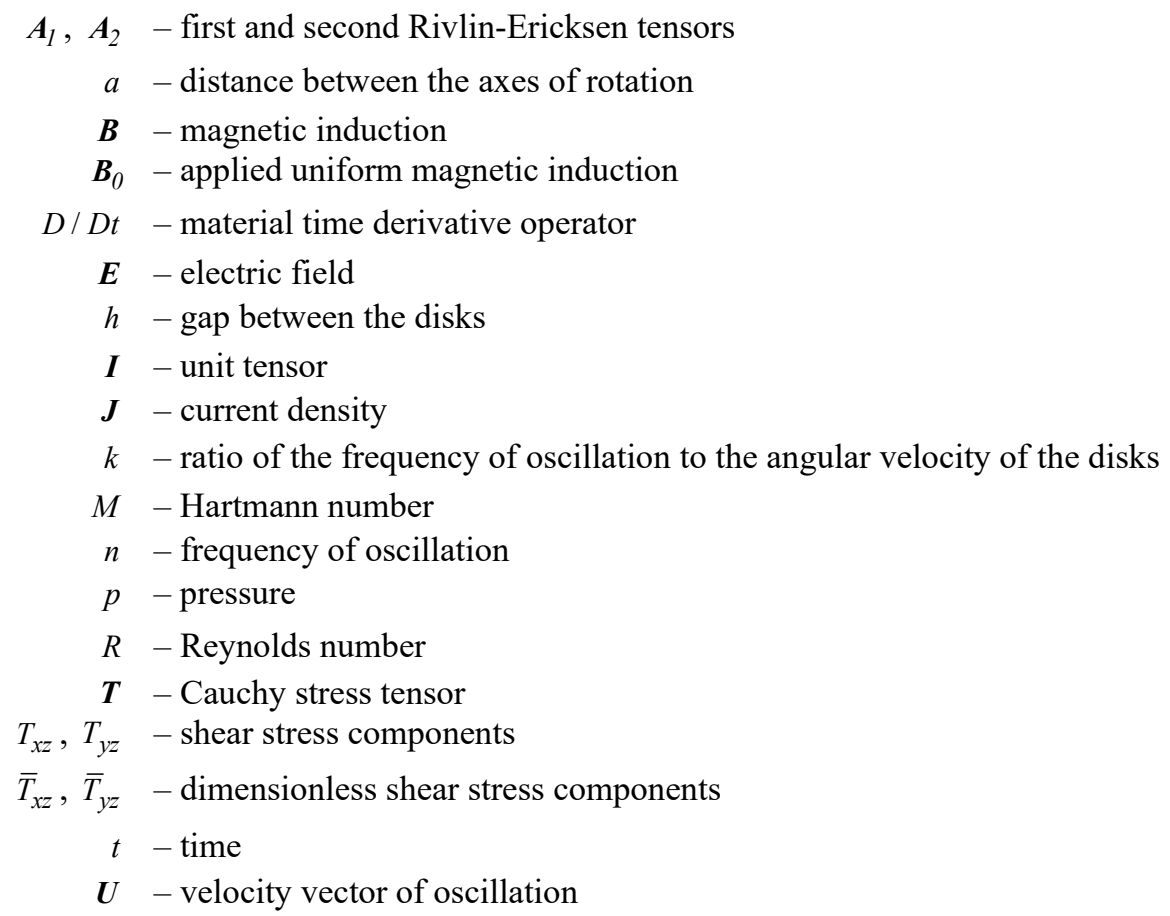




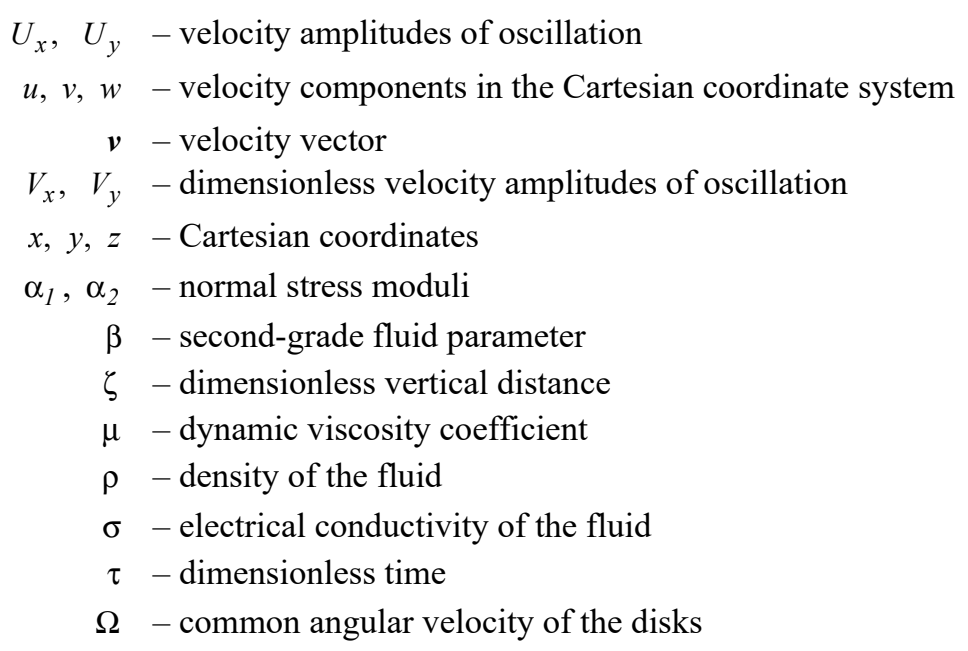

\section{References}

[1] Maxwell B. and Chartoff R.P. (1965): Studies of a polymer melt in an orthogonal rheometer.- Transactions of the Society of Rheology, vol.9, No.1, pp.41-52.

[2] Abbott T.N.G. and Walters K. (1970): Rheometrical flow systems - part 2: Theory for the orthogonal rheometer, including an exact solution of the Navier-Stokes equations.- Journal of Fluid Mechanics, vol.40, No.1, pp.205-213.

[3] Rajagopal K.R. and Gupta A.S. (1981): Flow and stability of a second grade fluid between two parallel plates rotating about noncoincident axes.- International Journal of Engineering Science, vol.19, No.11, pp.1401-1409.

[4] Rajagopal K.R. (1982): On the flow of a simple fluid in an orthogonal rheometer.- Archive for Rational Mechanics and Analysis, vol.79, No.1, pp.39-47.

[5] Rao A.R. and Rao P.R. (1985): MHD flow of a second grade fluid in an orthogonal rheometer.- International Journal of Engineering Science, vol.23, No.12, pp.1387-1395.

[6] Rajagopal K.R. (1992): Flow of viscoelastic fluids between rotating disks.- Theoretical and Computational Fluid Dynamics, vol.3, No.4, pp.185-206.

[7] Srinivasa A.R. (2000): Flow characteristics of a multiconfigurational, shear thinning viscoelastic fluid with particular reference to the orthogonal rheometer.- Theoretical and Computational Fluid Dynamics, vol.13, No.5, pp.305-325.

[8] Siddiqui A.M., Rana M.A. and Ahmed N. (2010): Magnetohydrodynamics flow of a Burgers' fluid in an orthogonal rheometer.- Applied Mathematical Modelling, vol.34, No.10, pp.2881-2892.

[9] Erdoğan M.E. (1999): Flow due to parallel disks rotating about non-coincident axis with one of them oscillating in its plane.- International Journal of Non-Linear Mechanics, vol.34, No.6, pp.1019-1030.

[10] Erdoğan M.E. (2000): Unsteady flow between two eccentric rotating disks executing nontorsional oscillations.International Journal of Non-Linear Mechanics, vol.35, No.4, pp.691-699.

[11] Ersoy H.V. (2012): Unsteady flow produced by oscillations of eccentric rotating disks.- Mathematical Problems in Engineering, Article ID 734784, pp.1-14.

[12] Giri A., Das S. and Jana R.N. (2014): Unsteady hydromagnetic flow due to oscillations of eccentric rotating disks.Journal of Nature Science and Sustainable Technology, vol.8, No.2, pp.237-258.

[13] Ersoy H.V. (2015): Periodic flow due to oscillations of eccentric rotating porous disks.- Advances in Mechanical Engineering, Article ID 1687814015599727, pp.1-8.

[14] Ersoy H.V. (2017): Periodic flow due to non-torsional oscillations of eccentric rotating porous disks in the presence of a magnetic field.- Mechanika, vol.23, No.3, pp.397-401.

[15] Ersoy H.V. (2018): Periodic flow of a second-grade fluid induced by non-torsional oscillations of eccentric rotating disks.- Sadhana - Academy Proceedings in Engineering Sciences, vol.43, Article No.36, pp.1-8. 
[16] Rivlin R.S. and Ericksen J. L. (1955): Stress-deformation relations for isotropic materials.- Journal of Rational Mechanics and Analysis, vol.4, pp.323-425.

[17] Dunn J.E. and Fosdick R.L. (1974): Thermodynamics, stability and boundedness of fluids of complexity 2 and fluids of second grade.- Archive for Rational Mechanics and Analysis, vol.56, No.3, pp.191-252.

[18] Dunn J.E. and Rajagopal K.R. (1995): Fluids of differential type: Critical review and thermodynamic analysis.International Journal of Engineering Science, vol.33, No.5, pp.689-729.

[19] Fosdick R.L. and Rajagopal K.R. (1979): Anomalous features in the model of second order fluids.- Archive for Rational Mechanics and Analysis, vol.70, No.2, pp.145-152.

Received: February 26, 2021

Revised: April 23, 2021 